\title{
Study On Stroke In Young Patient Due To Cardiac Disease in Tertiary Care Hospital in Dhaka City
}

\author{
SANJOY KUMAR SAHA, ${ }^{1}$ PRODIP KUMAR BISWAS, ${ }^{2}$ MEHERUNESSA MUKTA ${ }^{3}$ MOHAMMED SHAHADAT \\ HOSSAIN, ${ }^{4}$ MIMAL KUMAR BASU, ${ }^{5}$ FAHREEN RAHMAN, ${ }^{6}$ FERDOUS ALAM, ${ }^{7}$ ANWARUL KABIR ${ }^{8}$
}

\begin{abstract}
Objectives: Study of the risk factors associated with stoke due to cardiac disease in age group between 18 to 45 years of age,evaluate the risk factor of hospitalized stroke patients \& to search for cardiac disease associated with stroke also appropriate preventive measure applicable for our country.

Materials \& Methods: Non randomized prospective observational study was carried out in the department of Neurology, Medicine \& Cardiology unit of Dhaka Medical College Hospital between the periods of July 2009 to December 2009. Fifty (50) consecutive indoor patients more than 18 years of both sex admitted with suspicion of stroke. Diagnosis of stroke will be made from details clinical history clinical examination and confirmed by CT scan or MRI.

Result: Fifty patients were randomly chosen for this study, of which 26 (52\%) patients were male, 24 (48\%) patients were female \& male: female ratio was 1.08: I. Highest incidence of stroke was in between 3rd \& 4th decade. The incidence of ischemic stroke 46 (92\%) \& hemorrhagic stroke 4(8\%). Cardio embolism is the major (92\%) risk factor for ischemic stroke. Among the cardiac risk factors, valvular disease (64\%), MI (16\%), \&IHD (I0\%), Atrial fibrillation 8\%, Patent foramen ovale $2 \%$.Among the patients $33.33 \%$ of women with cerebral infarction were using OCP. Majority of the patients in this study were sedentary worker (45\%). Among the stroke patients $8 \%$ \& $10 \%$ had previous H/O stroke \& TIA respectively.

Conclusion: In this study a number of modifiable risk factors were identified, of which valvular heart disease \& HTN are important risk factor next are DM \& IHD smoking. Most of the valvular heart diseases are rheumatic in origin, which can be prevented through primary \& secondary prevention of rheumatic fever.
\end{abstract}

Key words: Stroke, Cardiac disease, Tertiary level hospital, Young patient

\section{Introduction}

Stroke is a clinical syndrome consisting of a constellation of neurological findings. Sudden or rapid in onset which persists for more than 24 hours \& whose vascular origins are limited to thrombotic or embolic occlusion of a cerebral artery resulting in infraction or spontaneous rupture of a vessel resulting in intracerebral or subarachnoid hemorrhage. Stroke in young adults may be devastating \& frequently no cause can be found. Though there are some overlapping in the risk factors between the some clearly distinct risk factors for ischemic \& hemorrhagic stroke in young adults, e.g. oral contraceptive pill, pregnancy, postpartum state, connective tissue diseases with vacuities, hematological variables, drug abuse, smoking, congenital heart diseases, family history, some genetic diseases etc. In terms of causation stroke in young adults is remarkably heterogeneous. Western reports $1,2,3$ show that in young age group, cardiac embolization \& non-atherosclerotic vasculopathies are rarely important cause of ischemic stroke as compared to atherosclerotic vasculopathy\& small artery occlusion. Stroke type in the young may influence the outcome \& may have a dramatic impact on the quality of life in survivors ${ }^{4}$. Diagnostic approach to stroke in young adults differs from that for older patients. A thorough investigation is recommended looking

1. Associate Professor (cc) of Medicine, Bashundhara Ad Din Medical College, Hasnabad, South Keranigonj, Dhaka.

2. Assistant Professor of Medicine, Dhaka Medical College, Dhaka.

3. Medical officer, Dhaka Community Medical College,Moghbazar,Dhaka

4. Assistant Professor of Medicine Faridpur Medical College

5. Senior Consultant Pathology, Pabna General Hospital.

6. Pathologist, 500 Bedded Mugda General Hospital, Dhaka.

7. Assistant Professor of Surgery Bangabandhu Sheikh Mujib Medical University

8. Professor, Gastroenterology, BSMMU

Address of correspondence: Dr. Sanjoy Kumar Saha, Associate Professor(cc), Department of Medicine, Bashundhara Ad Din medical College, Hasnabad, South Keranigonj, Dhaka. Mobile:01195326885, E-mail:sahasanjoy072@gmail.com 
into a broad array of potentials etiologies especially cardiovascular events. Cardioembolic sources are often occult so when the cause of ischemic stroke in young person is unclear after a thorough initials diagnostic evaluation, it is worthwhile to take a second look to the heart. The incident of stroke in Bangladesh is not known, it is about $10 \%$ of all emergency of stroke in medical words. However, prognosis of ischemic stroke to young adults is reported as favorable despite of its relationship with the presence of severity of complication at the time of the first event \& transient ischemic attack (TIA) are an important determinant of stroke, with 90 days risk of stroke reported as high as $10.5 \%$ \& the stroke risk apparent in first week. So the writing of the article aims at dissemination \& making alert doctors of all level for preventive measure \& early diagnosis \& therapeutic intervention due to cardiovascular diseases.

\section{Materials \& Method}

This is a non randomized prospective observational study was carried out in the department of Neurology, Medicine \& Cardiology unit of Dhaka Medical College Hospital between the period of July 2009 to December 2009. Fifty (50) consecutive patients 18 -45 years of age both sex admitted in Dhaka Medical College Hospital suspicion of stroke was included in the study. All routine investigation were done including $\mathrm{TC}, \mathrm{DC}, \mathrm{ESR}, \mathrm{Hb} \%$, total platelet count Urine for R/M/E, S. Creatinine, FBS\& 2HABF, CXR P/A view,ECG, Fasting Lipid profile, Anti Phospholipids antibody, Protein C \& S confirmed by CT scan or MRI of brain \& evaluation of cause due to cardiac diseases through appropriate relevant investigation like Echocardiography (Color Doppler/Trans esophageal). History of head injury, ICSOL, any demyelinating disease, bleeding disorder evident by history or by brain imaging \& Patients who die immediate after admission were excluded from the study. All the data were analyzed and presented by simple statistical percentage and tabulated form by SPSSsystem. Informed consent was taken from the patient or from the attendant of the patient for participation in the study. Patients were followed up during their stay in the hospital to observe the outcome. Ethical Clearance was taken from local medical ethical committee of Dhaka Medical College Hospital.

\section{Result}

Stroke incidence rises exponentially with increasing age. In this present study, all the patients were grouped in five age groups. Majority of the study subjects were above 41 years of age
Table-I

Demographic variation of stroke

\begin{tabular}{|c|c|}
\hline Variable & Frequency $(\%)$ \\
\hline \multicolumn{2}{|c|}{ Age distribution } \\
\hline $18-23$ & $2(4 \%)$ \\
\hline $24-29$ & $9(18 \%)$ \\
\hline $30-35$ & $10(20 \%)$ \\
\hline $36-40$ & $11(22 \%)$ \\
\hline $41-45$ & $18(36 \%)$ \\
\hline \multicolumn{2}{|c|}{ Sex distribution } \\
\hline Male & $26(52 \%)$ \\
\hline Female & $24(48 \%)$ \\
\hline \multicolumn{2}{|l|}{ Occupation } \\
\hline Cultivator & $3(6 \%)$ \\
\hline Day labour & $4(8 \%)$ \\
\hline Student & $4(16 \%)$ \\
\hline Unemployed & $8(16 \%)$ \\
\hline Businessman & $4(8 \%)$ \\
\hline House wife & $14(28 \%)$ \\
\hline Service & $6(12 \%)$ \\
\hline Others & $3(6 \%)$ \\
\hline \multicolumn{2}{|l|}{ Smoking habit } \\
\hline Nonsmoker & $10(20 \%)$ \\
\hline Smoker & $40(80 \%)$ \\
\hline \multicolumn{2}{|c|}{ Distribution of patients according residence } \\
\hline Urban & $28(56 \%)$ \\
\hline Rural & $22(44 \%)$ \\
\hline
\end{tabular}

Table- 1 shows that only $4 \%$ occurred in $<20$ years \& $36 \%$ in $41-45$ years. In this study $52 \%$ were male \& $48 \%$ were female i.e. male incidence is $8 \%$ higher than female $\&$ ratio is $1.08: 1$. In occupational category, service holder $12 \%$, businessman $8 \%$, housewife $28 \%$, student $16 \%$, laborer $8 \%$, unemployed $16 \%$. This study indicates that, those who were hard worker were affected less. The present study shows $20 \%$ patients were non smoker $\& 80 \%$ werecurrent smoker, incidence of stroke was more among smoker. The study shows that patients came from both urban $(56 \%) \&$ rural (44\%) were suffering from stroke. Urban population was more vulnerable. The study shows that the stroke incidence high in low income group (64\%) then middle income group $(32 \%)$. 
Table-II

\begin{tabular}{lc}
\hline Variable & Frequency (\%) \\
\hline State of patient during onset & $8(16 \%)$ \\
Sleep & $8(16 \%)$ \\
Work & $1(2 \%)$ \\
Excitement & $28(56 \%)$ \\
Rest & $5(10 \%)$ \\
Others & $4(8 \%)$ \\
Previous History & $5(10 \%)$ \\
Stroke & $41(82 \%)$ \\
Stroke with Hypertension(n-40) & \\
Previously Known & $16(66.67 \%)$ \\
Regularly treated & $6(25 \%)$ \\
Irregular/no treatment & $10(20 \%)$ \\
Diagnosed on Admission & $10(41.67 \%)$ \\
Family History(n-50) & $8(33.33 \%)$ \\
Diabetes mellitus & \\
Hypertension & \\
\hline Stroke & \\
\hline & \\
Nistory & $(16 \%)$ \\
\hline
\end{tabular}

Table-II shows that majority of the stroke occurs at rest $(56 \%)$. The study shows $8 \%$ of the patient had previous history of stroke. $10 \%$ of the patient had previous history of TIA. In the observed patients regarding previous $20 \%$ of the were ex smoker, $8 \%$ of the patients had the habit of betel nut chewing,Only $2 \%$ had H/O alcohol consumption. Study shows majority $58.33 \%$ of the stroke suffered female patients never used oral contraceptive in their life time.33.33\% was regularly taking oral contraceptive. That among the hypertensive patient only $25 \%$ were regularly treated, $41.67 \%$ were on irregular or no treatment. A good number of patient $33.33 \%$ was diagnosed as hypertensive patient for the first time after hospital admission. The study regarding family history shows that about $32 \%$ of patient has history of stroke, $20 \%$ hypertension, 16\% Diabetes mellitus, $12 \%$ Ischemic heart disease.
Table-III

\begin{tabular}{lc}
\hline Variable & Frequency (\%) \\
\hline Frequency of different heart diseases & $8(16 \%)$ \\
a)Myocardial infarction & \\
Anterior & 6 \\
Inferior & 2 \\
b)Ischemic heart diseases & $5(10 \%)$ \\
Anterior ischemia & 1 \\
Inferior ischemia & 4 \\
c) valvular heart disease & $32(64 \%)$ \\
Mitral stenosis with mitral regurtation & $7(14 \%)$ \\
(MS with MR) & \\
Mitral stenosis (MS) & $24(48 \%)$ \\
Mitral stenosis with Aortic stenosis & $1(2 \%)$ \\
(MS with AS) & \\
d) Atrial fibrillation & $4(8 \%)$ \\
e) Patent foramen ovale & $1(2 \%)$ \\
CT Scan findings (n-50) & \\
Ischemic & $4(8 \%)$ \\
Intracerebral Haemorrhage (ICH) &
\end{tabular}

Outcome of stroke patients during discharge (n-50) (Accoding to Barthel Index)

Improved 38(76\%)

Static $\quad 8(16 \%)$

Deteriorated $4(8 \%)$

In this study cardiogenic cerebral embolus is one of the most common causes of ischemic stroke in the young due to cardiac disease accounting for unto $92 \%$ of cases. Among the cardio embolic cause, the mitral valvular disease is the most common. In this study transthoracic echocardiography was done in fifty patients \& transoesophageal echocardiography was done in six patients. There was only one patent foramen ovale, detected by doing transoesophageal echocardiography which was missed by transthoracic echocardiography. Most of the patients with valvular heart disease had H/O Rheumatic Fever. Mitral valvular heart diseases were more prevalent in this study group. Among them most of the patients were female. 19 patient had left atrial mural thrombus with vegetation, $8 \%$ had atrial fibrillation \& Left anterior ventricular wall \& inferior wall was found hypo kinetic in $1 \& 4$ patients respectively. CT \& MRI findings of the studied patients show 
that $92 \%$ had ischemic stroke while $8 \%$ had hemorrhagic stroke due cardiac disease. According to Barthel Index during discharge $76 \%$ of the patient was improved, $16 \%$ were static \& the remaining $8 \%$ were deteriorated.

\section{Discussion}

This study was observational retrospective hospital based $\&$ was carried out to determine the risk factors for stroke in young adults (18-45 years) in hospitalized patient in tertiary care hospital of Dhaka city during the period from July 2009 to December 2009. Risk factors for stroke among these patients were identified \& correlated. Stroke incidence rises exponentially with increasing age. ${ }^{7}$ In this study, all the patients were grouped in five age groups. Majority of the study subjects were above 41 years of age. Bevan etal ${ }^{8}$ in his study of stroke in young adults also found similar picture Bell et al (1990) studied 50 patients with stroke. Most of the incidence of stroke was between the ages of 50-69 years.In this study (table-1) $52 \%$ were male \& $48 \%$ were female i.e. male incidence is $8 \%$ higher than female \& ratio is $1.08: 1$ which coincides with international study. The present study coincides with study of Chowdhuryet $11^{10} \&$ is slightly higher than the finding of Kurtzke ${ }^{11}$ where showed that frequency of stroke is $30 \%$ higher in men than women.CT \& MRI findings of the studied patients show (table-3) that $92 \%$ had ischemic stroke while $8 \%$ had hemorrhagic stroke. This study similar with study of Alam B et a1, ${ }^{12}$ they studied 1020 patients of stroke in $\mathrm{DMCH}$. In their study the incidence of ischemic stroke was $93 \%$ \& hemorrhagic stroke was $7 \%$. Higher rate of hemorrhagic stroke also have been reported in number of hospital series in Asian countries such as Singapore, Malaysia (33\%), Thailand (30\%), Korea (31\%), Taiwan (31\%). Higher rate of hemorrhagic stroke in this present hospital based study may be due to the acute admission is more related to the hemorrhagic stroke.

In this study cardiogenic cerebral embolus is one of the most common causes of ischemic stroke in the young due to cardiac disease accounting for unto $92 \%$ of cases. Among the cardio embolic cause, the mitral valvular disease is the most common. In this study transthoracic echocardiography was done in fifty patients \&transoesophageal echocardiography was done in six patients. There was only one patent foramen ovale, detected by doing transoesophageal echocardiography which was missed by transthoracic echocardiography. There might be more patent foramen ovals, if TEE could be done in the young adults group, whose risk factor was not identified. Most of the patients with valvular heart disease had H/O Rheumatic Fever. Mitral valvular heart diseases were more prevalent in this study group. Among them most of the patients were female. Bevan et al ${ }^{14}$ shows that about $35.4 \%$ cerebral infarcts are due to cardioembolic source. Among 24 mitral valvular heart diseases, 19 patient had left atrial mural thrombus with vegetation\& only 4 patient had atrial fibrillation most likely due to rheumatic valvular heart disease.In this study there were cardiac risk factors, other than valvular heart disease. Anterior ventricular wall \& inferior wall was found hypokinetic in $1 \& 4$ patients respectively on transthoracic echocardiography. Among them $16 \%$ patients had H/O MI \& 10\% patients had IHD. This study shows that coronary artery disease in earlier age is also a risk factor ischemic stroke.

Present study shows that $16.67 \%$ of stroke patients were suffering from HTN. Bevan et $\mathrm{al}^{14}$ in "Stroke in young adults" showed that $31 \%$ of the patient with cerebral infarction had HTN. This present study correlates with this western study. This study shows that $33.33 \%$ stroke patients did not aware that they were hypertensive \& $25 \%$ were on irregular treatment. This present study is almost similar with the study of Chowdhury et al, ${ }^{10}$ who studied 74 hypertensive patients who suffered stroke $\&$ had shown that $34 \%$ of the patients were not aware that they were hypertensive \& $60.7 \%$ were on irregular treatment. You canary et $\mathrm{al}^{15}$ showed in his study that the percentage of ischemic stroke was similar with normal tensile patients but the incidence was higher in untreated cases of HTN. So elective control of HTN is essential.The present study shows $20 \%$ patients were smoker. Multiple individual studies have demonstrated that the risk of stroke is increased among the cigarette smokers. Yano et al \& Donanet $a{ }^{16}$ have shown strong association between cigarette smoking \& stroke. Similar study in Copenhagen \& Finland" 19 has shown increase risk of stroke in smoker. It has been reported by New Zealand researcher 208 that passive smoking significantly increases the risk of stroke among the nonsmoker \& in long term ex-smoker.An analysis from 32 studies, ${ }^{17}$ found that relative risk of ischemic stroke for smoker were 1.9 times more hand that of non-smoker. In this study out of 24 female patients, $58.33 \%$ patient had no $\mathrm{H} / \mathrm{O}$ taking OCP, $33.33 \%$ of patient was currently taking OCP. In this series $33.33 \%$ of the women with cerebral infarction were using OCP when their stroke occurred, only $8.33 \%$ had strokes attributable solely to OCP. It must be remembered that pregnancy increases the risk of ischemic events by approximately 13 times. Four women among our young patients experienced strokes ( 2 hemorrhagic, 2 ischemic) during the period of pregnancy \& pure premium. Physical activity has inverse relationship with the stroke. Exercise is effective in the prevention of cardiovascular disease \& stroke. The benefits are largely manifested through the role that exercise plays an important 
role in control of certain modifiable risk factors such as blood lipid abnormalities, diabetes \& obesity. This study shows that $20 \%$ of the study subjects were heavy workers, $35 \%$ were moderate workers, $45 \%$ sedentary workers. This study indicates that, those who were hard worker were affected less. Herman et al ${ }^{17}$ suggested that physical activity reduces the risk of stroke. In this present study sedentary workers form the major percentage of stroke. The present study shows, only $8 \%$ patients had previous $\mathrm{H} / \mathrm{O}$ of stroke \& only $10 \%$ had previous H/O TIA. An western study shows that $18 \%$ (Aho et al) \& 26\% (walker et $\mathrm{al}^{1}$ ) patients suffered acute stroke had past $\mathrm{H} / \mathrm{O}$ one or more episode of stroke which is higher than this present study. In this study, only $16 \%$ of the patients were in sleep, $56 \%$ were resting state during attack $\&$ the rest of the patients were awake. It suggested acute mental stress is either a precipitant of stroke or an important underlying cause. Hayeeet $\mathrm{al}^{13}$ found in his study that $61 \%$ of his study subjects were suffered from acute stressful events before the attack of stroke.

Finally out of 50 patients, the conditions of 38 patients were improved, 8 patients were static \& 4 patients were deteriorated.

\section{Conclusion}

Stroke is one of the foremost causes of morbidity, mortality $\&$ a socioeconomic challenge, more so in Bangladesh where health system including the rehabilitation is not within the reach of ordinary people. It is crystal clear that, this devastating condition not only affects the patient but also their family. There are many risk factors for stroke, some are modifiable \& some are not. In this study a number of modifiable risk factors were identified, of which valvular heart disease \& HTN are important risk factor next is DM, IHD, smoking. Stroke is more preventable than to cure. In an under developed country like ours, the best policy for combating stroke is primary prevention. Most of the valvular heart diseases are rheumatic in origin, which can be prevented through primary \& secondary prevention of rheumatic fever. Extensive investigations arenecessary to find out etiology ofyoung adults other than cardiac diseases \& aggressive treatment \& secondary prevention as well as rehabilitation should be considered.

\section{Conflict of Interest : None}

\section{References:}

1. Wallker AE Robins M, Weinfeld FD. Clinical findings. Stroke 1981;12:115

2. Walker B. Alam M, Habib S, Hossain B, Haque A, Haque et. Prevalence of Stroke in Bangladeshi populationpopulation based Study. JAFMC Bangladesh.In press 2009.

3. Kristensen B, Malm J, Carberg B, Stegmayr B, Backman G, Fagerlung M, et al. Epidemiology \& etiology of ischemic
Stroke in young adults aged 18-44 Yrs in Northern Sweden. Stroke Bangladesh 1997;28:1702-9.

4. Bogousslavsky J, Peierre P. Ischemic Stroke under age 45. Neurolclin 1992;10:113-24.

5. Hachinski V, Norris JW. The young Stroke, in the acute Stroke. Contemporary neurology series 1985;27:141-163.

6. Nencni P, Inzitari D, Baruffi MC. Incidence of Stroke in young adults in Florence, Italy. Stroke 1988;19:977-981.

7. Botania R, Beaglegore R, North J. Events, incidence \& case fatality rates of, cerebrovascular disease in AUCKLAND, New Zealand. Am J Epidemiology 1984;120:236-43.

8. H Bevan, K Sharma, W Bradly. Stoke In Young Adults. Stoke 1990;21:382-386.

9. Bell DA, William Brien, Vladimar II, keefe BO. Antiphospholipidsynd : Prevalence among patients with Stoke \&TIA.Am J Med 1990;88:593-97.

10. Chowdhury SZM. Study of Risk Factors in Cerebrovascular Disease- A study of 100 case (Dissertation). Dhaka: BCPS, 1991:48.

11. Kurzke JF. Epidemiology of cerebrovascular disease. In: P. Rowland L, editor. Merrtt's Neurology. Philadelphia: LL W; 2000:135-176.

12. Alam B, Mohammd Q D, Habib M, Hossain S, Haque A, et al. Stroke -Evaluation of Risk Factors. Bangladesh Journal of Neuroscience 1999;15(2):14-18.

13. Hayee MA, Anwarullah AKM, Haque A, AKhtar N. Analysis of Risk factors of Stroke in 472 Cases. Bangladesh Journal of Neuroscience 1998;14(2):41-54.

14. H Bevan, K Sharma, W Bardly. Stroke In Young Adults. Stroke 1990;21:382-386.

15. Yoshinnari M, Kaku R, Iwase M, Lino K, Yamamato M, Satou Y, et al. Development of ischemic Stroke in normotensive \& hypertensive treatment: 8 Year follow up study. J- Diabetes- Complication 1997;11(10):9-14.

16. Donnan AG, Dignin H. Smoking as a risk factor for cerebral ischaemia Lancet 1949;16:643434-7.

17. Herman B. Leyten ACM, Van Luijk JH, Frenken CWGM, Low AAW, Schulle BPM . An elevation of risk factors for Stroke in a Duch Community. Stroke 1982;13:334-39.

18. Hart CL, Smith GD, Hole DJ, Hawthorne VM. Alcohol consumption \& mortality from all cases, coronary heart disease \& Stroke result from a prospective cohort study of Scottish men with 21 years of follow up. BMJ; 1999;318:1725-29.

19. Boysen G, Nyboe J, Appleyard M, Sorensen PS, Boas JH, Edward M Stroke incidence \& risk factors \& Stroke in Copenhagen, Denmark. Stroke 1988;19:1345-53. 